\title{
Suppression of lipid peroxidation in adrenal microsomes following ACTH administration to guinea pigs
}

\author{
J M Burczynski, J R Hayes, J M Voigt, P A Longhurst \\ and $\mathbf{H}$ D Colby
}

Albany College of Pharmacy, 106 New Scotland Avenue, Albany, New York 12208, USA

(Requests for offprints should be addressed to H D Colby; Email: colbyh@acp.edu)

\begin{abstract}
Previous studies demonstrated high levels of lipid peroxidation (LP) in the guinea pig adrenal cortex. The present studies were done to determine if adrenal LP activity was influenced by $\mathrm{ACTH}$, the major hormonal regulator of the gland. Guinea pigs were treated with ACTH for 1, 3 or 7 days. In addition, some guinea pigs received ACTH for 7 days and were killed 3 or 7 days later. After treatment, adrenal microsomal fractions were prepared and incubated in vitro with $1 \mathrm{mM}$ ferrous sulfate to initiate LP. ACTH treatment caused a progressive decrease in adrenal LP; activity was almost totally inhibited within 3 days. The inhibitory effects of ACTH on LP were dose-dependent. Following cessation of ACTH treatment, adrenal LP gradually returned toward control levels. Microsomal con-
\end{abstract}

centrations of linoleic acid, a major substrate for adrenal $\mathrm{LP}$, were increased by ACTH administration and then also returned to control levels after cessation of treatment. There were no significant changes in adrenal $\alpha$-tocopherol or $\beta$-carotene concentrations resulting from ACTH treatment. The results indicate that ACTH has a role in the regulation of adrenal LP. The actions of ACTH cannot be attributed to an increase in adrenal content of the antioxidants, $\alpha$-tocopherol and $\beta$-carotene, or to a decrease in LP substrate. The actions of ACTH to inhibit LP may contribute to an increase in adrenal hormone production by protecting steroidogenic enzymes from peroxidative degradation.

Journal of Endocrinology (2001) 168, 333-338

\section{Introduction}

Lipid peroxidation (LP) has been extensively investigated in a variety of tissues because of its putative role in a number of pathological and physiological processes (Tribble et al. 1987, Girotti 1998). It has been proposed that the adrenal cortex is likely to be one of the organs most affected by LP, in part because of its high content of unsaturated fatty acids, the substrates for LP (Hornsby \& Crivello 1983, Hornsby 1989). In addition, the mitochondrial and microsomal P450 enzymes that catalyze steroidogenesis in the adrenal cortex are sources of oxygen radicals that may initiate LP. It is also known that LP can effect the degradation of adrenal P450 cytochromes, thereby compromising steroidogenic enzyme activities (Wang \& Kimura 1976, Imataka et al. 1985, Takayanagi et al. 1986, Staats et al. 1988a). Thus, LP has the potential to modulate the production of adrenocortical hormones, but a physiological role has yet to be definitively established.

Prior investigations demonstrated high levels of LP in various adrenal membrane preparations in vitro (Kitabchi 1967, Wang \& Kimura 1976, Imataka et al. 1985, Staats \& Colby 1987, Staats et al. 1988b, 1989). In addition, extensive deposition of lipofuscin, a byproduct of LP, has been found in adrenal glands (Belloni et al. 1987, Cheng et al. 1996), suggesting the occurrence of LP in vivo as well. Interestingly, the accumulation of lipofuscin increases with aging (Reichel 1968, Cheng et al. 1996, Almeida et al. 1998), coincident with a decline in adrenal androgen secretion (Adams 1985, Parker 1991), but a cause-andeffect relationship has not been established. If LP has a role in the control of adrenal hormone production, regulatory mechanisms might be anticipated. The studies presented in this report were done to determine if the major hormonal modulator of adrenocortical steroidogenesis, adrenocorticotropic hormone (ACTH), also participates in the regulation of adrenal LP.

\section{Materials and Methods}

\section{Animals}

Adult male Strain 2 guinea pigs were maintained under standardized conditions of light (0600-1800 h) and temperature $\left(22^{\circ} \mathrm{C}\right)$ with freely available food and water. All animals were allowed at least 2 weeks to become acclimated to the housing conditions prior to use in experiments. Experimental animals were treated with ACTH 
(Acthar gel, Rhone-Poulenc Rorer Inc., Collegeville, PA, USA, $20 \mathrm{IU} / \mathrm{kg}$ body weight, twice daily) by s.c. injection for 1,3 or 7 days and then killed by $\mathrm{CO}_{2}$ inhalation. Additional animals were treated with the same dose of ACTH for 7 days and killed 3 or 7 days following the last treatment to evaluate the reversibility of ACTH actions. In a dose-response experiment, guinea pigs received $2 \cdot 5,10$ or $20 \mathrm{IU}$ ACTH for 7 days and were then killed by $\mathrm{CO}_{2}$ inhalation. Adrenal glands were quickly removed, trimmed free of adhering connective tissue and fat, frozen in liquid nitrogen, and stored at $-75{ }^{\circ} \mathrm{C}$ until the preparation of microsomal fractions.

\section{Tissue preparation}

Adrenal glands were homogenized in $1.15 \% \mathrm{KCl}$ in $0.05 \mathrm{M}$ Tris $-\mathrm{HCl}(\mathrm{pH} \mathrm{7.4})$ at a tissue concentration of $12.5 \mathrm{mg} / \mathrm{ml}$. Subcellular fractions were prepared by differential centrifugation as described previously (Eacho \& Colby 1985). Microsomal pellets were then resuspended in KCl-Tris buffer at a concentration of $2.5 \mathrm{mg} / \mathrm{ml}$ (wet weight equivalent).

\section{$L P$}

Microsomal suspensions $(2.5 \mathrm{ml})$ were placed in $25 \mathrm{ml}$ Erlenmeyer flasks and incubated at $37^{\circ} \mathrm{C}$. $\mathrm{FeSO}_{4}$, at a final concentration of $1.0 \mathrm{mM}$, was added to initiate LP. One set of flasks was kept on ice to serve as an unincubated or 0 -time control. Two $100 \mu \mathrm{l}$ aliquots were removed from each flask after 15, 30 and $60 \mathrm{~min}$ of incubation, and placed in microcentrifuge tubes containing $30 \mu \mathrm{l} 40 \%$ trichloroacetic acid on ice to precipitate protein and inhibit further LP. Samples were then centrifuged for 2 min in a microcentrifuge to fully precipitate the protein pellet. One hundred microliters of supernatant were added to $0.75 \mathrm{ml}$ of $1 \%$ thiobarbituric acid (TBA) and incubated at $90{ }^{\circ} \mathrm{C}$ for $30 \mathrm{~min}$. Tubes were then placed on ice for $10 \mathrm{~min}$ prior to extraction of TBA-reactive substances (TBARS) into n-butanol. The amount of TBARS in each sample was calculated from the fluorescence intensity at an excitation wavelength of $532 \mathrm{~nm}$ and an emission wavelength of $553 \mathrm{~nm}$, using malondialdehyde (MDA) as the standard. Thus, TBARS are calculated as MDA equivalents and expressed as $\mathrm{pmol} / \mathrm{mg}$ wet weight equivalent of whole adrenal gland.

\section{a-Tocopherol}

$\alpha$-Tocopherol was extracted from microsomal suspensions with hexane in the presence of butylated hydroxytoluene (BHT) as described by Lang et al. (1986); $\gamma$-tocopherol was added as an internal standard. The hexane extracts were dried under a stream of nitrogen and reconstituted in $200 \mu \mathrm{l}$ ethanol. Aliquots were analyzed with a Waters HPLC system (Milford, MA, USA) as described pre- viously (Feingold et al. 1993) using fluorescence detection with an excitation wavelength of $292 \mathrm{~nm}$ and an emission wavelength of $340 \mathrm{~nm}$. Values are expressed as $\mathrm{ng} / \mathrm{mg}$ wet weight equivalent of whole adrenal gland.

\section{$\beta$-Carotene}

Microsomal $\beta$-carotene concentrations were determined as described by Newcomb et al. (1990). Microsomal suspensions were extracted with hexane in the presence of BHT; $\beta$-apo- $8^{\prime}$-carotenal was used as an internal standard. Aliquots of the extract were analyzed with a Waters HPLC system using UV detection at $450 \mathrm{~nm}$ to quantify $\beta$-carotene.

\section{Fatty acid analyses}

Microsomal total fatty acid concentrations were determined by modification of the method described previously (Staats et al. 1988b, 1989). Briefly, microsomal suspensions were hydrolyzed and total fatty acids were extracted into hexane in the presence of BHT. Cholesteryl n-octanoate $(5 \cdot 0 \mu \mathrm{g})$ was added to each sample as an internal standard to estimate recoveries. Hexane extracts were dried under nitrogen and samples were trimethylsilyated before injection into the gas chromatograph (GC). GC analyses were done on a Hewlett Packard 5890 Series II GC equipped with a single flame ionization detector set at $360{ }^{\circ} \mathrm{C}$, using helium as make-up gas, and a Grob-type split injection port $(\sim 16: 1$ ratio $)$ set at $270{ }^{\circ} \mathrm{C}$. A Hewlett Packard (Palo Alto, CA, USA) HP-1 column $(30 \mathrm{~m} \times 0.32 \mathrm{~mm}$, $0 \cdot 1 \mathrm{~mm}$ film thickness) was used with helium as the carrier gas. The column conditions were set at $170{ }^{\circ} \mathrm{C}$ for $2 \mathrm{~min}$, increased by $5{ }^{\circ} \mathrm{C} / \mathrm{min}$ to $200{ }^{\circ} \mathrm{C}$, followed by $10{ }^{\circ} \mathrm{C} / \mathrm{min}$ to $290^{\circ} \mathrm{C}$. Fatty acids were identified by co-injection of known fatty acid standards and quantified using the internal standard.

\section{Data analyses}

All data are presented as means \pm S.E.M. and were analyzed by Student's $t$-test or Newman-Keuls multiple range test, as appropriate. Each 'experiment' constitutes the results obtained with adrenal glands from an individual animal and thus represents an independent sample. Most of the microsomal data are expressed per equivalent wet weight of tissue. However, the results are similar and the conclusions equally applicable when expressed per milligram microsomal protein.

\section{Results}

Administration of ACTH to guinea pigs for 7 days caused a dose-dependent decrease in $\mathrm{Fe}^{2+}$-induced LP in adrenal microsomes (Fig. 1). Similar results were obtained when 


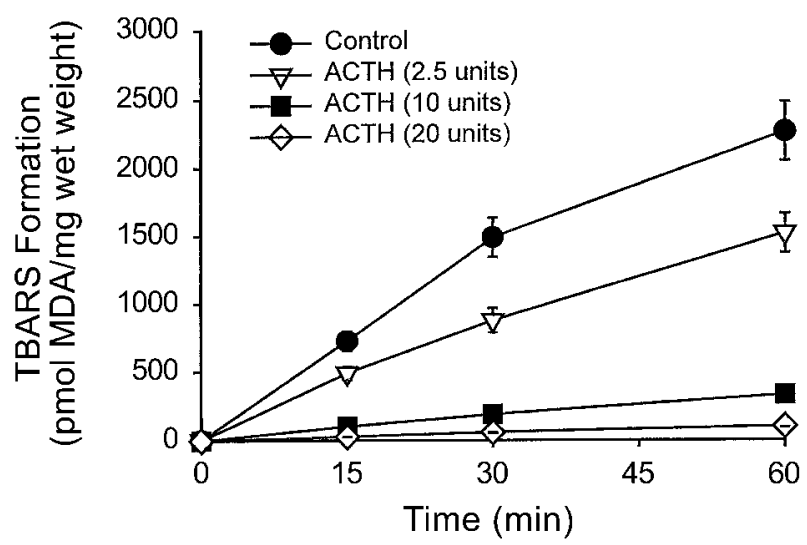

Figure 1 Dose-response effects of ACTH treatment for 7 days on adrenal microsomal TBARS formation during incubation with $1.0 \mathrm{mM}$ ferrous ion. Values are the means \pm S.E.M. of four to six animals per group. $P<0 \cdot 05$ for all $\mathrm{ACTH}$ groups vs corresponding control values.

incubations were done with adrenal mitochondrial preparations in place of microsomes (data not shown). Doses of ACTH as low as $2.5 \mathrm{IU} / \mathrm{kg}$ twice a day significantly decreased TBARS formation at each of the incubation times studied; the two higher doses caused almost complete inhibition of LP (Fig. 1). Each of the doses of ACTH employed caused increases in adrenal gland weights and thus should be considered supraphysiological. The increases in adrenal mass ranged from approximately $10 \%$ at the lowest dose to approximately $40 \%$ at the highest dose.

Time-course studies using ACTH at a dose of $20 \mathrm{IU} / \mathrm{kg}$ twice a day revealed significant inhibition of adrenal microsomal LP within 1 day (Fig. 2). After 3 days, inhibition of LP by ACTH was greater than $90 \%$, and the inhibition was sustained through 7 days of ACTH treat-

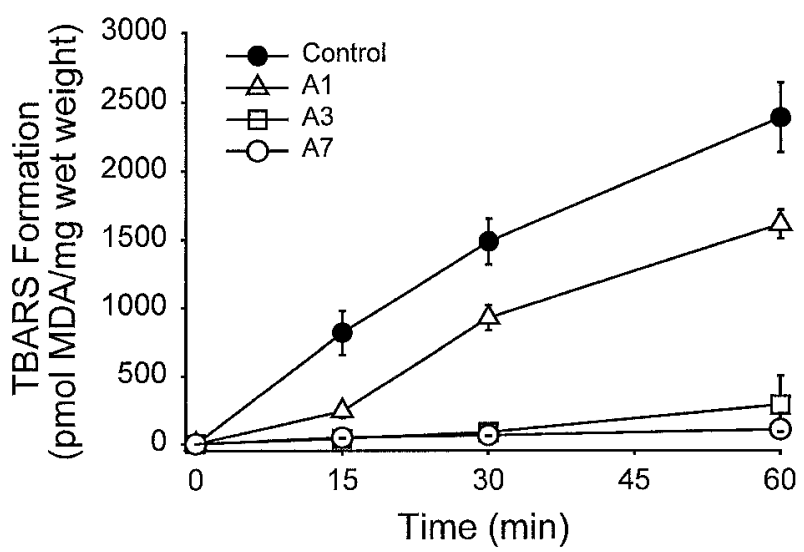

Figure 2 Time-course for the effects of ACTH treatment $(20 \mathrm{IU} / \mathrm{kg}$ twice a day) for 1 (A1), 3 (A3) and 7 (A7) days on adrenal microsomal TBARS formation during incubation with $1.0 \mathrm{mM}$ ferrous ion. Values are means \pm S.E.M. of four animals per group. $P<0.05$ for all ACTH groups vs corresponding control values.

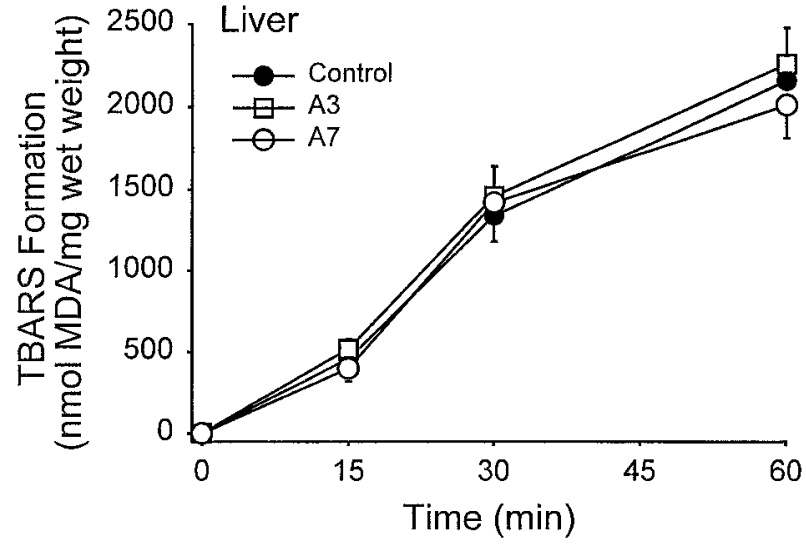

Figure 3 Absence of effects of ACTH treatment (20 IU/kg twice a day) for 3 (A3) or 7 (A7) days on hepatic microsomal TBARS formation during incubation with $1.0 \mathrm{mM}$ ferrous ion. Values are means \pm S.E.M. of four animals per group.

ment (Fig. 2). As expected, because liver cells lack ACTH receptors, ACTH treatment had no effect on TBARS formation by hepatic microsomes (Fig. 3) that were obtained from the same animals.

To determine the reversibility of ACTH effects on adrenal LP, guinea pigs were treated with ACTH for 7 days and then killed 3 or 7 days after the last treatment. As shown in Fig. 4, after 3 days without exogenous ACTH, production of TBARS by adrenal microsomes increased significantly but remained below control values. At 7 days after ACTH treatment, TBARS formation increased further to within approximately $25 \%$ of control values (Fig. 4).

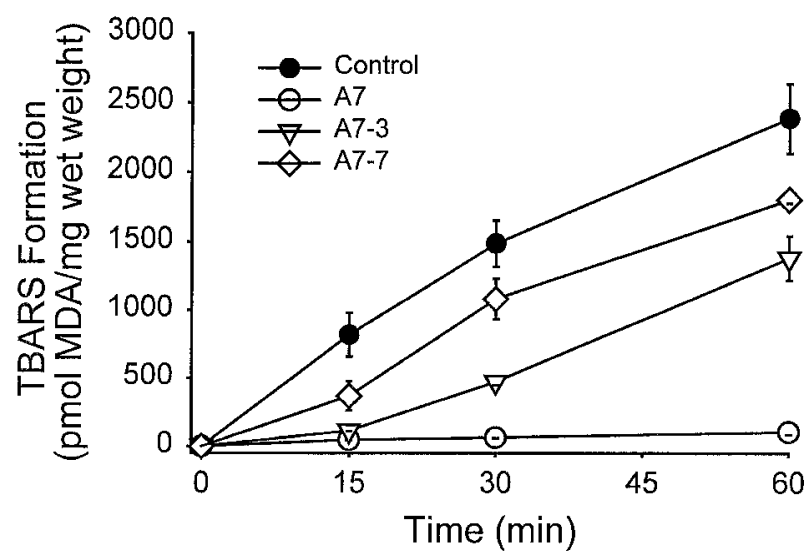

Figure 4 Reversibility of the effects of ACTH treatment on TBARS formation by adrenal microsomes during incubation with $1.0 \mathrm{mM}$ ferrous ion. Animals received ACTH (20 IU/kg twice a day) for 7 days and were killed immediately (A7), 3 days later (A7-3) or 7 days later $(A 7-7)$. Values are means \pm S.E.M. of four animals per group. $P<0.05$ for all $\mathrm{ACTH}$ groups vs corresponding control values. 


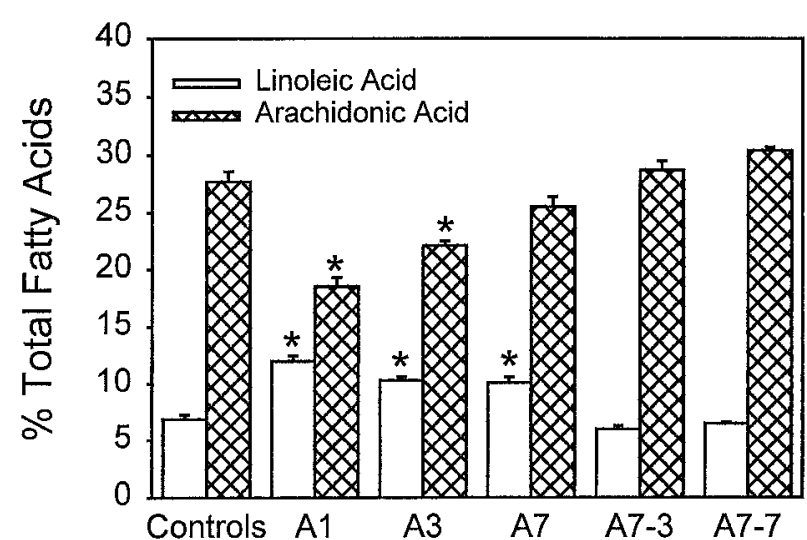

Figure 5 Time-course and reversibility of the effects of ACTH treatment $(20 \mathrm{IU} / \mathrm{kg}$ twice a day) on the relative concentrations of linoleic and arachidonic acid in adrenal microsomes. Values are the means \pm S.E.M. of four animals per group. ${ }^{*} P<0 \cdot 05$ (vs corresponding control value). Abbreviations as in legends to Figs 2 and 4 .

Accompanying the effects of ACTH on adrenal LP were changes in the total fatty acid profiles of adrenal microsomes (Fig. 5). The relative amounts of linoleic acid, one of the preferred substrates for LP, significantly increased following ACTH treatment for 1, 3 and 7 days; values returned to control levels after termination of ACTH administration. In contrast, the relative content of arachidonic acid in adrenal microsomes decreased by approximately $30 \%$ after 1 day of ACTH treatment, but returned to control levels by 7 days. The amounts of the other major fatty acids found in guinea pig adrenal microsomes (palmitic, stearic, oleic) were not significantly affected by ACTH treatment.

To determine if the effects of ACTH on adrenal LP were mediated by changes in membrane antioxidant concentrations, adrenal microsomal concentrations of $\alpha$-tocopherol and $\beta$-carotene were measured. ACTH treatment tended to increase microsomal $\alpha$-tocopherol concentrations (Fig. 6), but the changes were not statistically significant. In addition, there were no significant changes in microsomal $\beta$-carotene concentrations resulting from ACTH treatment (not shown). For example, microsomal $\beta$-carotene concentrations in control and 7-day ACTH-treated animals were $393 \pm 65$ and $407 \pm 52 \mathrm{pmol} / \mathrm{g}$ equivalent wet weight (means \pm S.E.M., $n=4$ per group) respectively.

\section{Discussion}

The adrenal cortex contains very high concentrations of several antioxidants, most notably $\alpha$-tocopherol and ascorbic acid (Hornsby \& Crivello 1983, Staats \& Colby 1987, Feingold et al. 1993). Nonetheless, both in vitro observations (Staats \& Colby 1987, Staats et al. 1988b, 1989) and

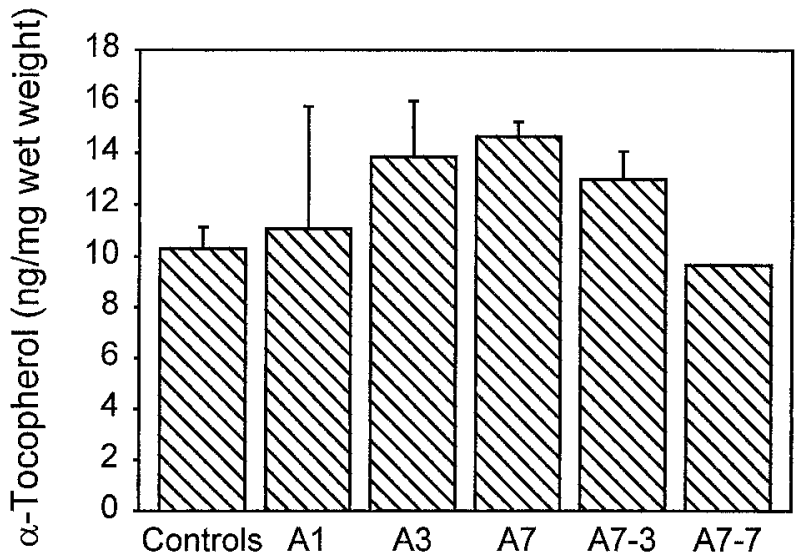

Figure 6 Time-course and reversibility of the effects of ACTH treatment $(20 \mathrm{lU} / \mathrm{kg}$ twice a day) on adrenal microsomal concentrations of $\alpha$-tocopherol. Values are means \pm S.E.M. of four animals per group. Abbreviations as in legends to Figs 2 and 4 .

reports on the accumulation of lipofuscin in adrenals in vivo (Reichel 1968, Belloni et al. 1987, Cheng et al. 1996, Almeida et al. 1998) suggest high levels of LP in the gland. As noted by others, the large amounts of polyunsaturated fatty acids found in adrenocortical membranes may predispose the adrenals to LP. In addition, it is possible that the free radical production associated with the function of P450-catalyzed steroidogenic enzymes serves as an ongoing stimulus for the initiation of LP (Hornsby \& Crivello 1983, Hornsby 1989). Thus, despite its extensive antioxidant protection, other factors may overcome these defenses and make the adrenal cortex susceptible to peroxidative activity.

The physiological significance of LP in the adrenal cortex, if any, remains uncertain. A number of laboratories have demonstrated adverse effects of LP on adrenocortical function (Kitabchi 1967, Kitabchi \& West 1975, Wang \& Kimura 1976, Brogan et al. 1983, Klimek et al. 1983, Imataka et al. 1985). It has been demonstrated that hormone synthesis is compromised and LP is high in $\alpha$-tocopherol-deficient animals (Kitabchi 1980), but little is known about the mechanisms responsible for the functional changes. We have found that there are large regional differences in LP in the guinea pig adrenal cortex that are inversely related to some steroid hydroxylase activities in each zone (Staats \& Colby 1987, Staats et al. 1988a). Kitabchi (1967) and Kitabchi \& West (1975) reported a relationship between ascorbate-induced LP and a decline in microsomal steroid hydroxylase activities. Similarly, Wang \& Kimura (1976) and Klimek et al. (1983) found that LP promoted the degradation of cytochrome P450 in bovine adrenal mitochondria. Our own observations (Brogan et al. 1983) as well as studies by Imataka et al. (1985) also indicate that LP causes a decline in adrenal steroid hydroxylase activities and that there are differences in the sensitivities of various adrenal enzymes to the effects 
of LP. The 17 $\alpha$-hydroxylase/C17,20-lyase reactions that are catalyzed by $\mathrm{P} 450 \mathrm{c} 17$ seem to be particularly vulnerable to inactivation by LP (Imataka et al. 1985). Such differences in enzyme sensitivity, in combination with regional differences in LP, may contribute to the uniqueness of enzyme activities in each of the zones of the adrenal cortex.

Indirect evidence suggests that LP is an ongoing process in the adrenal cortex and thus potentially of functional significance. As noted above, the extensive deposition of lipofuscin granules in the adrenal cortex of humans and other species (Hornsby \& Crivello 1983, Cheng et al. 1996) is consistent with high levels of LP. Interestingly, adrenal accumulation of lipofuscin increases with aging and is greatest in the zona reticularis of the gland, the same zone that is most susceptible to LP in vitro (Staats \& Colby 1987, Staats et al. 1988b, 1989). The zona reticularis is also the major source of adrenal androgens, whose secretion decreases markedly with aging (Adams 1985, Parker 1991). These observations suggest a possible cause-andeffect relationship between LP and adrenal androgen secretion that seems worthy of further investigation.

Adrenocortical secretion of glucocorticoids and androgens is controlled principally by the pituitary hormone, ACTH. The actions of ACTH to stimulate steroidogenesis include acute effects on substrate mobilization and longterm effects on the synthesis of steroidogenic enzymes (Waterman 1994, Parker \& Schimmer 1995). Inhibition of LP by ACTH, as reported herein, may further enhance steroidogenesis by protection of adrenal cytochromes $\mathrm{P} 450$ from peroxidative degradation. In addition, studies by Kimura (1986) demonstrated stimulatory effects of unsaturated phospholipids on adrenal steroidogenesis. Thus, inhibition of LP by ACTH might serve to both decrease adrenal enzyme inactivation and preserve lipids that are needed for optimal steroidogenic activity. The magnitude of its effects and the relatively rapid reversibility of ACTH actions on LP may be indicative of hormonal regulation that is physiological in nature.

It is possible that the actions of ACTH on LP are mediated by increases in adrenal glucocorticoid and/or androgen secretion. However, we have found that addition of steroids in vitro to adrenal microsomal or mitochondrial preparations does not inhibit $\mathrm{Fe}^{2+}$-induced LP. Similarly, administration of glucocorticoids or androgens to guinea pigs for up to 7 days does not mimic the effects of ACTH treatment on adrenal LP (H D Colby, unpublished observations). Nonetheless, these approaches may not adequately simulate the changes in intra-adrenal steroid concentrations that result from ACTH administration and accordingly, a role for adrenal steroids in the inhibition of LP cannot at present be excluded.

Although our findings demonstrate marked suppression of adrenal LP in vitro following ACTH administration to guinea pigs, the in vivo relevance is uncertain. However, the observations of Cheng et al. (1996) that ACTH treatment decreases the concentration of lipofuscin in rat adrenal glands suggests a decline in LP in vivo as well. Also, in our studies, the effect of ACTH to increase adrenal concentrations of linoleic acid may be explained by inhibition of LP in vivo. Prior studies (Kitabchi 1967) as well as our own observations indicate that linoleic and arachidonic acids are the principal substrates for LP in adrenal membranes. The initiation of LP in adrenal microsomes in vitro selectively decreases the concentrations of these two fatty acids. In addition, $\alpha$-tocopherol deficiency in guinea pigs increases adrenal LP activity and decreases microsomal and mitochondrial concentrations of linoleic and arachidonic acids (Staats et al. 1988a, 1989). Thus, since high levels of LP are associated with losses of adrenal linoleic acid, the increases in linoleic acid caused by ACTH treatment (Fig. 5) are consistent with a decline in LP in vivo.

Inhibition of LP by ACTH might also be expected to increase arachidonic acid levels in adrenal membranes if peroxidative turnover decreases in vivo. However, ACTH has other direct effects on the release and metabolism of adrenal arachidonic acid related to its putative role as a mediator of steroidogenesis (Solano et al. 1988, Yamazaki et al. 1996). Therefore, accelerated metabolism of arachidonic acid along alternative pathways may obscure the impact of a decline in LP on membrane concentrations (Solano et al. 1988, Yamazaki et al. 1996). In any case, arachidonic acid concentrations in adrenal microsomes returned to control levels after 7 days of ACTH treatment and linoleic acid concentrations remained elevated (Fig. 5), but LP in vitro was almost completely suppressed at that time. Thus, the inhibition of LP by ACTH cannot readily be attributed to a deficiency of fatty acid substrate.

Further investigations are now needed to determine the mechanism of action of ACTH on adrenal LP. Although the data are not included in this report, ACTH inhibited LP in adrenal mitochondria as well as in microsomes. The changes produced in mitochondrial LP were quantitatively very similar to those in microsomes. Since the effects of ACTH treatment in vivo are demonstrable in isolated microsomal and mitochondrial preparations in vitro, changes must be produced directly within adrenal membranes. High concentrations of ascorbic acid are found in adrenal cytosol, but none is detected in microsomes or mitochondria (Staats et al. 1988a, 1989), excluding a role for this antioxidant in the effects of ACTH on LP. We previously found that in vitro adrenal LP was highly correlated (inversely) with membrane $\alpha$-tocopherol concentrations under a variety of experimental conditions (Staats \& Colby 1987, Staats et al. 1988a,b, 1989, Burczynski et al. 1999), and speculated that ACTH treatment increased adrenal $\alpha$-tocopherol content, thereby inhibiting LP. However, the experimental results obtained do not support this hypothesis. In addition, the concentrations of another membrane antioxidant, $\beta$-carotene, were also not significantly affected by ACTH treatment. It 
is possible that ACTH effects a redistribution of antioxidant(s) within adrenal membranes to enhance their effectiveness. Alternatively, changes in adrenal lipid profiles and/or membrane fluidity may alter the capacity for initiation or propagation of LP. Studies on these and other potential mechanisms should contribute to a fuller understanding of the factors affecting LP in the adrenal cortex and may provide new insight into the functional significance of LP in the gland.

\section{References}

Adams JB 1985 Control of secretion and the function of C19-delta 5 -steroids of the human adrenal gland. Molecular and Cellular Endocrinology 41 1-17.

Almeida H, Magalhaes MC \& Magalhaes MM 1998 Age-related changes in lipid peroxidation products in rat adrenal gland. Age $\mathbf{2 1}$ 119-121.

Belloni AS, Mazzocchi G, Manteroi F \& Nussdorfer GG 1987 The human adrenal cortex: ultrastructure and base-line morphometric data. Journal of Submicroscopic Cytology and Pathology 19 657-668.

Brogan WC, Miles PR \& Colby HD 1983 Effects of lipid peroxidation on adrenal microsomal monooxygenases. Biochimica et Biophysica Acta 758 114-120.

Burczynski JM, Hayes JR, Longhurst PA \& Colby HD 1999 Species differences in adrenal lipid peroxidation: role of $\alpha$-tocopherol. Free Radical Biology and Medicine 26 987-991.

Cheng B, Tserng KY \& Kowal J 1996 Characterization and identification of an adrenal age-related nonpolar fluorescent substance. Endocrinology 137 2447-2456.

Eacho PI \& Colby HD 1985 Differences in microsomal steroid metabolism between the inner and outer zones of the guinea pig adrenal cortex. Endocrinology 116 536-541.

Feingold IB, Longhurst PA \& Colby HD 1993 Regulation of adrenal and hepatic $\alpha$-tocopherol content by androgens and estrogens. Biochimica et Biophysica Acta 1176 192-196.

Girotti AW 1998 Lipid hydroperoxide generation, turnover, and effector action in biological systems. Journal of Lipid Research 39 1529-1542.

Hornsby PJ 1989 Steroid and xenobiotic effects on the adrenal cortex: mediation by oxidative and other mechanisms. Free Radical Biology and Medicine 6 103-115.

Hornsby PJ \& Crivello JF 1983 The role of lipid peroxidation and biological antioxidants in the function of the adrenal cortex. Part 2. Molecular and Cellular Endocrinology 30 123-147.

Imataka H, Suzuki K \& Tamaoki B 1985 Effect of Fe induced lipid peroxidation upon microsomal steroidogenic enzyme activities of porcine adrenal cortex. Biochemical and Biophysical Research Communications 128 657-663.

Kimura T 1986 Transduction of ACTH signal from plasma membrane to mitochondria in adrenocortical steroidogenesis. Effects of peptide, phospholipid, and calcium. Journal of Steroid Biochemistry 25 711-716.

Kitabchi AE 1967 Inhibition of steroid C-21 hydroxylase by ascorbate: alterations of microsomal lipids in beef adrenal cortex. Steroids $\mathbf{1 0}$ $567-576$.

Kitabchi AE 1980 Hormonal status in vitamin E deficiency. In Vitamin E, a Comprehensive Treatise, pp 348-371. Ed. LJ Machlin. New York: Marcel Dekker.
Kitabchi AE \& West WH 1975 Effect of steroidogenesis on ascorbic acid content and uptake in isolated adrenal cells. Annals of the New York Academy of Sciences 258 422-431.

Klimek J, Schaap AP \& Kimura T 1983 Effect of paraquat on cytochrome P450-dependent lipid peroxidation in bovine adrenal cortex mitochondria. Biochimica et Biophysica Acta 752 127-136.

Lang JK, Gohil K \& Packer L 1986 Simultaneous determination of tocopherols, ubiquinols, and ubiquinones in blood, plasma, tissue homogenates and subcellular fractions. Analytical Biochemistry 157 106-116.

Newcomb SA, Culling-Berglund AJ \& Davis TP 1990 Endogenous levels of $\beta$-carotene in human buccal mucosa cells by reversed-phase high-performance liquid chromatography. Journal of Chromatography A $\mathbf{5 2 6}$ 47-50.

Parker KL \& Schimmer BP 1995 Transcriptional regulation of the genes encoding the cytochrome P450 steroid hydroxylases. Vitamins and Hormones 51 339-370.

Parker LN 1991 Control of adrenal androgen secretion. Endocrinology and Metabolism Clinics of North America 20 71-83.

Reichel WJ 1968 Lipofuscin pigment accumulation and distribution in five rat organs as a function of age. Journal of Gerontology 23 145-153.

Solano AR, Dada L \& Podesta EJ 1988 Lipoxygenase products as common intermediates in cyclic AMP-dependent and -independent adrenal steroidogenesis in rats. Journal of Molecular Endocrinology 1 $147-154$.

Staats DA \& Colby HD 1987 Regional differences in microsomal lipid peroxidation and antioxidant levels in the guinea pig adrenal cortex. Journal of Steroid Biochemistry and Molecular Biology 28 637-642.

Staats DA, Lohr DP \& Colby HD 1988a Effects of tocopherol depletion on the regional differences in adrenal microsomal lipid peroxidation and steroid metabolism. Endocrinology 123 975-980.

Staats DA, Lohr D \& Colby HD 19886 Relationship between mitochondrial lipid peroxidation and alpha-tocopherol levels in the guinea-pig adrenal cortex. Biochimica et Biophysica Acta 961 279-284.

Staats DA, Lohr DP \& Colby HD 1989 - -Tocopherol depletion eliminates the regional differences in adrenal mitochondrial lipid peroxidation. Molecular and Cellular Endocrinology 62 189-195.

Takayanagi R, Kato K \& lbayashi H 1986 Relative inactivation of steroidogenic enzyme activities of in vitro vitamin E-depleted human adrenal microsomes by lipid peroxidation. Endocrinology 119 464-469.

Tribble DL, Aw TY \& Jones DP 1987 The pathophysiological significance of lipid peroxidation in oxidative cell injury. Hepatology $7377-386$.

Wang HP \& Kimura T 1976 Ferrous ion-mediated cytochrome P450 degradation and lipid peroxidation in adrenal cortex mitochondria. Biochimica et Biophysica Acta 423 374-381.

Waterman MR 1994 Biochemical diversity of cAMP-dependent transcription of steroid hydroxylase genes in the adrenal cortex. Journal of Biological Chemistry $26927783-27786$.

Yamazaki Y, Higuchi K, Kominami S \& Takemori S 1996 15-Lipoxygenase metabolite(s) of arachidonic acid mediates adrenocorticotropin action in bovine adrenal steroidogenesis. Endocrinology 137 2670-2675.

Received 26 May 2000

Accepted 4 October 2000 